\title{
ÉTUDE SUR LA BIOLOGIE DES PREMIERS STADES LARVAIRES \\ D'ANGUILLICOLA CRASSUS (NEMATODA, DRACUNCULOIDEA) : SPÉCIFICITÉ DE L'HÔTE INTERMÉDIAIRE ET INFLUENCE DE LA TEMPÉRATURE SUR LA DURÉE DU DÉVELOPPEMENT
}

\author{
S. BONNEAU*, G. BLANC** et Annie J. PETTER*
}

\footnotetext{
* Laboratoire des Vers, Muséum National d'Histoire Naturelle, 61 rue de Buffon, 75231 PARIS Cedex 05.

** Service d'Aquaculture et Pathologie Aquacole, Ecole Nationale Vétérinaire de Nantes, C.P. 3013, 44087 NANTES Cedex 03 (les demandes de tirés à part sont à faire auprès de cet auteur).
}

\section{RÉSUMÉ}

Des tentatives d'infestation de divers Arthropodes avec des œufs et des larves écloses d'Anguillicola crassus, Nématode parasite de la vessie gazeuse de l'Anguille, sont effectuées. Le développement jusqu'au $3^{\ominus}$ stade est obtenu uniquement chez les Copépodes Cyclopidae etchez l'Ostracode Cypria ophthalmica. La croissance des larves et la durée du développement chez les Cyclopidae sont étudiées à 4 températures différentes : les premières larves du $3^{e}$ stade apparaissent à $\mathrm{J} 6$ à $28{ }^{\circ} \mathrm{C}$, à $\mathrm{J} 9$ à $21^{\circ} \mathrm{C}$ et à $\mathrm{J} 31$ à $12-14{ }^{\circ} \mathrm{C}$; à une température de 4-6 ${ }^{\circ} \mathrm{C}$ les larves ne montrent aucun développement après 91 jours d'infestation.

Mots-clés : Anguillicola crassus ; Nematoda ; Anguilla anguilla ; biologie des premiers stades larvaires.

\section{STUDIES ON THE BIOLOGY OF THE EARLY LARVAL STAGES OF ANGUILLICOLA CRASSUS (DRACUNCULOIDEA, NEMATODA) : SPECIFICITY OF THE INTERMEDIATE HOST AND EFFECT OF TEMPERATURE ON RATE OF DEVELOPMENT.}

\begin{abstract}
Infection of various Arthropods was attempted with eggs and larvae of Anguillicola crassus, a nematode parasite of the swimbladder of eels. Development up to the third larval stage was obtained only in Cyclopid Copepods and in Cypria ophthalmica (Ostracoda). Development was followed in Cyclopidae at 4 different temperatures : third-stage larvae first appeared on day 6 at $28{ }^{\circ} \mathrm{C}$, on day 9 at $21{ }^{\circ} \mathrm{C}$, and on day 31 at $12-14{ }^{\circ} \mathrm{C}$. No development occurred at $4-6{ }^{\circ} \mathrm{C}$ when observation was discontinued 91 days post infection.
\end{abstract}

Key-words : Anguillicola crassus ; Nematoda ; Anguilla anguilla ; biology of early larval stages.

\section{INTRODUCTION}

Depuis l'introduction il y a quelques années et la rapide extension de l'espèce Anguillicola crassus chez les anguilles européennes (Anguilla anguilla L.), de nombreux 
travaux ont été effectués aussi bien sur l'action pathogène de ces Nématodes (BLANC, 1989 ; BLANC et al., 1990) que sur leur biologie et leur cycle évolutif complet a été réalisé en laboratoire (De CHARLEROY et al., 1990 ; BONNEAU, 1990). Cependant, la plupart des expériences d'infestation de l'hôte intermédiaire ont été réalisées avec des Copépodes (ainsi, DE CHARLEROY et al., 1987, ont obtenu l'infestation de 10 espèces de Cyclopidae, alors que 2 espèces d'Harpacticoida ne se sont pas infestées), et peu d'auteurs ont tenté l'infestation d'autres Invertébrés.

Par ailleurs, le développement des larves chez les Cyclopidae a en général été réalisé à une température avoisinant $21^{\circ} \mathrm{C}$, température à laquelle le $3^{e}$ stade est atteint après 10 à 13 jours (PETTER et al., 1989 ; DE CHARLEROY et al., 1990). Les variations de la durée du développement en fonction de la température n'ont été étudiées que par quelques observations préliminaires : PETTER et al., 1990 , ont constaté que des $3^{\text {es }}$ stades apparaissent dès le $6^{\circ}$ jour d'infestation quand la température varie de $18{ }^{\circ} \mathrm{C}$ la nuit à $29{ }^{\circ} \mathrm{C}$ dans l'après-midi, et par contre ont observé l'ébauche de la $3^{\mathrm{e}}$ mue seulement après 62 jours d'infestation à $12^{\circ} \mathrm{C}$.

Nous avons ici, d'une part, cherché à vérifier si d'autres Arthropodes intervenant dans le régime alimentaire des Anguilles, en particulier des Insectes adultes et larvaires qui n'ont jamais été testés, pouvaient jouer le rôle d'hôtes intermédiaires, d'autre part, étudié la durée du développement des larves à différentes températures.

\section{MATÉRIEL ET MÉTHODES}

\section{Origine du matériel}

Les œufs d'Anguillicola crassus proviennent des utérus de femelles mûres recueillies, d'une part, dans la vessie gazeuse d'Anguilles jaunes provenant d'étangs du littoral méditerranéen et, d'autre part, dans la vessie gazeuse d'Anguilles argentées originaires d'étangs du Nord de la France et conservées au Laboratoire de Physiologie du Muséum National d'Histoire Naturelle de Paris.

La plupart des Arthropodes dulçaquicoles proviennent de mares, étangs et ruisseaux de la région parisienne, de la région de Bourges (Cher) et du voisinage de Nantes (Lac de Grand-Lieu, Loire Atlantique). Les Copépodes utilisés pour l'étude de la durée du développement à différentes températures proviennent d'une mare d'un jardin de Lagny (Seine-et-Marne) et du Lac de Grand-Lieu ; ils appartiennent à plusieurs espèces de Cyclopidae mélangées dans les 2 cas. d'Oléron).

Les Copépodes marins et d'eaux saumâtres proviennent de la Perrotine (lle

\section{Méthodes d'infestation}

Les Arthropodes sont placés dans de petits cristallisoirs dont le fond est tapissé d'œufs et de larves écloses d'Anguillicola crassus.

\section{- Tentatives d'infestation d'Invertébrés}

Les expériences sont effectuées à la température du laboratoire, variant de 18 à $22{ }^{\circ} \mathrm{C}$; les Arthropodes sont examinés après le $13^{\mathrm{e}}$ jour d'infestation, date à laquelle les premiers $3^{\text {es }}$ stades commencent à apparaître chez les Cyclopidae.

\section{- Etude de la durée du développement à différentes températures}

Les Cyclopidae ont été placés à 4 températures différentes :

- à $28{ }^{\circ} \mathrm{C}$ dans un insectarium à température contrôlée ; des Cyclopidae (1 à 4) sont examinés tous les jours jusqu'à l'apparition des premiers $3^{\text {es }}$ stades larvaires. 
- à $21^{\circ} \mathrm{C} \pm 0,5$ dans une étuve à température programmée, 10 Cyclopidae sont examinés chaque jour à heure fixe jusqu'à l'apparition des premiers $3^{\text {es }}$ stades larvaires.

- à $12-14{ }^{\circ} \mathrm{C}$ dans une étude à température programmée ; des Cyclopidae sont examinés à des intervalles de 1 à 4 jours du $10^{\mathrm{e}}$ au $32^{\mathrm{e}}$ jour d'infestation.

- à 4-6 ${ }^{\circ} \mathrm{C}$ dans un réfrigérateur : des Cyclopidae sont examinés tous les 6 à 8 jours jusqu'au $91^{\mathrm{e}}$ jour d'infestation.

\section{RÉSULTATS}

\section{Tentatives d'infestation d'Invertébrés}

Les résultats des tentatives d'infestation sont présentés dans le tableau I. Des larves du $3^{e}$ stade ont été observées uniquement chez les Cypria ophthalmica, ce qui avait déjà été observé par PETTER et al., 1990.

Chez les larves de Chironomidae, des larves du $2^{e}$ stade encore entourées de la mue du 1 er stade sont trouvées dans le tube digestif de certains spécimens.

Chez tous les autres Arthropodes examinés, aucune larve n'a été trouvée.

Tableau I : Tentatives d'infestation de divers Arthropodes par des larves L2 d'Anguillicola crassus ; observation du troisième stade larvaire.

Table I : Attempts to infect various Arthropods with L2 larvae of Anguillicola crassus ; observation of the third larval stage.

\begin{tabular}{ccc}
\hline & & NOMBRE \\
& NOMBRE & D'INDIVIDUS \\
HÔTE & D'INDIVIDUS & PARASITÉS PAR DES L3 \\
& EXAMINÉS & APRĖS 13 JOURS \\
& & D'INFESTATION \\
\hline
\end{tabular}

COPEPODES MARINS OU D'EAUX

SAUMATRES :

Cyclopinoides littoralis G. S. Brady

(Cyclopoida, Cyclopinidae)

Copépodes Harpacticoides benthiques

non identifiés

CLADOCERES :

Cerodaphnia laticauda Müller

OSTRACODES :

Cypria ophtalmica Jurine

expérience $\mathrm{N}^{\circ} 1$

expérience $\mathrm{N}^{\circ} 2$

AMPHIPODES :

Gammarus pulex L. (adultes)

ISOPODES :

Asellus aquaticus $\mathrm{L}$.

COLEOPTERES :

Hydroporus sp. 


\section{Durée du développement à différentes températures}

Le développement des larves aux différentes températures est représenté sur la figure 1 ; les Cyclopidae ayant été laissés en contact avec les larves pendant toute la durée de certaines expériences, nous ne pouvons savoir la date exacte à laquelle chaque larve a été ingérée ; seule, la plus grande larve recueillie chaque jour a donc été figurée.

A $28{ }^{\circ} \mathrm{C}$, la première L3 est observée à J6 (4 Copépodes examinés, un $3^{e}$ stade et six $2^{\text {es }}$ stades) ; à $\mathrm{J7}$, la plus grande partie des larves est au $3^{\mathrm{e}}$ stade $(3$ Copépodes examinés, quatre $3^{\text {es }}$ stades et un $2^{\mathrm{e}}$ stade).

A $21^{\circ} \mathrm{C}$, les premières L3 apparaissent à $\mathrm{J} 9$ (10 Copépodes examinés, vingt $3^{\text {es }}$ stades et trente-quatre $2^{\text {es }}$ stades de longueurs variées) ; à J10 la plus grande partie des larves est au $3^{\text {e }}$ stade (10 Copépodes examinés, trente-huit $3^{\text {es }}$ stades et six $2^{\text {es }}$ stades de longueurs variées).

A $12.14{ }^{\circ} \mathrm{C}$, les premières L3 apparaissent à J31 (4 Copépodes examinés, onze $3^{\text {es }}$ stades dont cinq sont encore entourés de la mue du $2^{\mathrm{e}}$ stade, et dix $2^{\text {es }}$ stades de longueurs variées) ; à $\mathrm{J} 32$, un Copépode examiné contient trois $3^{\text {es }}$ stades et quatre $2^{\text {es }}$ stades de longueurs variées.

A 4-6 ${ }^{\circ} \mathrm{C}$, les larves restent vivantes mais nous n'avons observé aucune croissance appréciable jusqu'au $91^{\mathrm{e}}$ jour d'infestation.
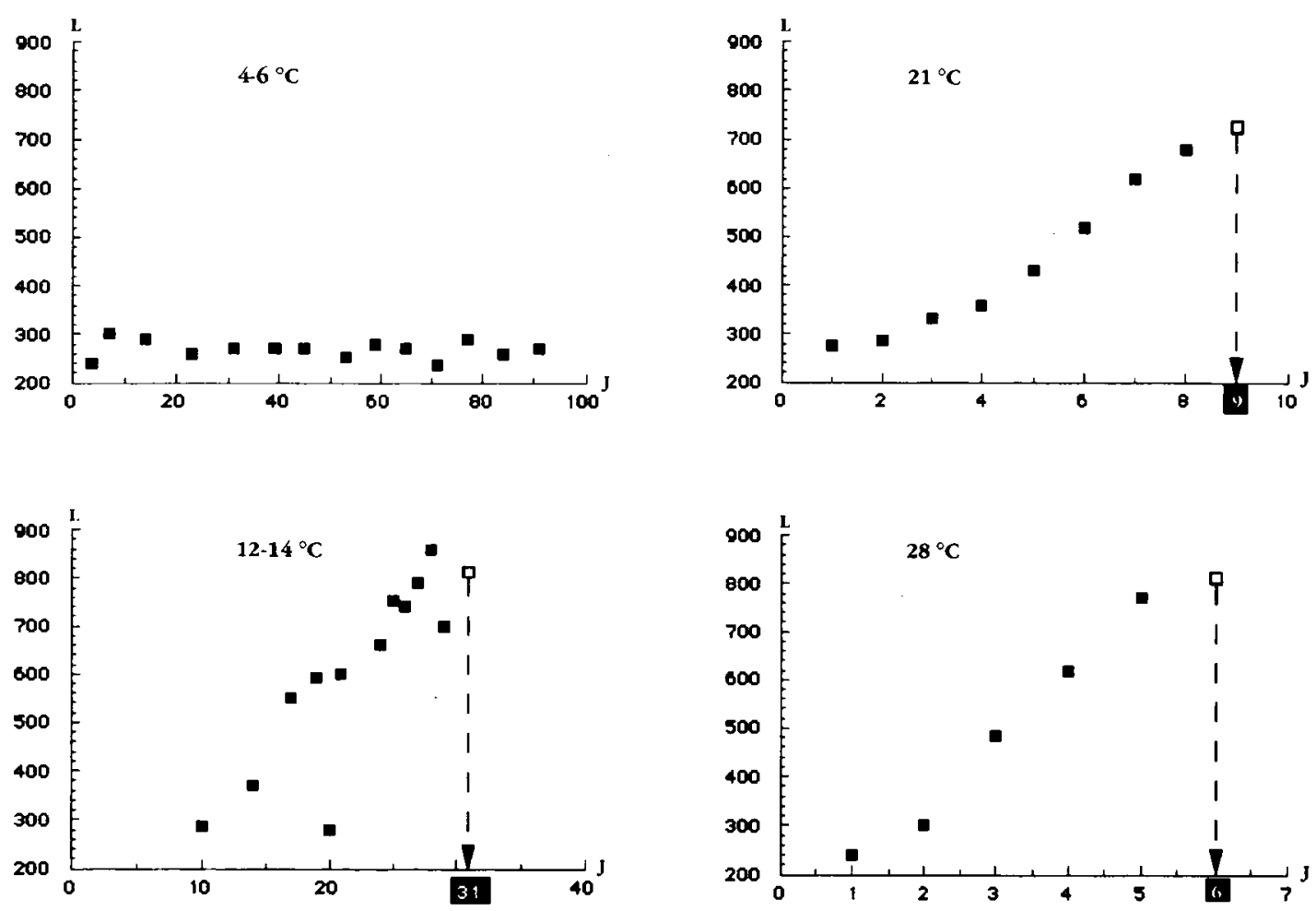

figure 1 : Développement larvaire d'Anguillicola crassus chez les Cyclopidae à différentes températures.

$J$ : Nombre de jours d'infestation. $\mathbf{L}$ : Longueur des larves $(\mu \mathrm{m})$. Carré noir $: 2^{e}$ stade. Carré blanc $: 3^{e}$ stade. (Seule la plus grande larve recueillie chaque jour a été figurée).

figure 1 : Development of Anguillicola crassus larvae in Cyclopid copepods at different temperatures.

$J$ : Number of days post infestation. $\mathbf{L}$ : Length of the larvae $(\mu \mathrm{m})$. Black spot : $2^{\text {nd }}$ stage larvae or molting larvae. White spot : third larval stage. (Each spot corresponds to the longest larva among those daily collected). 


\section{DISCUSSION}

\section{Spécificité de l'hôte intermédiaire}

Quelques tentatives d'infestation d'Arthropodes autres que les Cyclopidae par des larves d'Anguillicola crassus ont été effectuées:

HIROSE et al., (1976) ont observé que les larves ingérées par le Copépode Calanoida Sinodiaptomus chaffanjoni pénètrent dans la cavité générale, mais aucun Copépode parasité n'est retrouvé 24 heures après l'infestation.

DE CHARLEROY et al., (1987) ont tenté l'infestation de 2 genres de Copépodes Harpacticoida (Canthocampus sp. et Attheyala sp.) et n'ont pas observé de spécimens parasités après 3 jours.

KENNEDY et FITCH (1990) ont obtenu des résultats négatifs dans leurs essais d'infestation d'Asellus aquaticus, de Daphnia sp. et des Gammarus pulex adultes, alors que $50 \%$ des Gammarus pulex juvéniles, $8,7 \%$ des Diaptomus gracilis (Calanoida) et un Eurytemora affinis (Calanoida d'eau saumâtre) ont été trouvés infestés après 48 heures ; cependant, ces auteurs n'ont pas suivi le développement ultérieur des larves.

Le développement jusqu'au $3^{3}$ stade n'a donc actuellement été réalisé en dehors des Copépodes Cyclopidae que chez les Ostracodes de l'espèce Cypria opthalmica.

Certains Arthropodes d'autres groupes sont capables de s'infester, mais dans le cas du Calanoidea Sinodiaptomus chaffanjoni et des larves de Chironomidae, il a été constaté que les larves ne se développaient pas, et dans les autres cas (Amphipodes juvéniles et Copépodes Calanoida) le développement n'a pas été suivi au-delà de 48 heures.

II semble que les Daphnies (Cladocères), les Aselles (Isopodes), les Gammares adultes (Amphipodes), les Coléoptères et les larves de Diptères et d'Odonates, qui constituent une part importante du régime alimentaire des Anguilles (Tesch, 1977), ne s'infestent pas. Les quelques tentatives d'infestation de Copépodes Harpacticoida et de Cyclopinidae (Cyclopoida) sont également négatives.

Les Cyclopidae sont donc bien les principaux hôtes intermédiaires de l'espèce ; cependant, le fait que le développement puisse s'effectuer chez des Ostracodes semble indiquer une large spécificité, et il n'est pas exclu que ce développement soit également possible chez d'autres Crustacés dont le régime alimentaire permet l'ingestion des larves.

\section{Influence de la température sur la durée du développement}

PETTER et al., (1990) avaient observé qu'à $12{ }^{\circ} \mathrm{C}$ la croissance des larves était très faible pendant les 45 premiers jours, et que l'ébauche de la $2^{\mathrm{e}}$ mue n'apparaissait qu'à J62. Dans l'expérience exposée ci-dessous, réalisée dans la même étuve, la croissance des larves a été beaucoup plus rapide et les $3^{\text {es }}$ stades sont apparus à $\mathrm{J} 31$; la température de l'étuve présentant de légères fluctuations et n'ayant pas été vérifée chaque jour, nous supposons que cette différence est due à une température moyenne légèrement plus élevée dans la seconde expérience. Des expériences ultérieures devraient permettre d'expliquer ces variations.

Le développement à $28^{\circ} \mathrm{C}$ est 2 fois plus rapide qu'à la température du laboratoire, variant entre 18 et $22{ }^{\circ} \mathrm{C}$, et 5 fois plus rapide qu'à $12-14{ }^{\circ} \mathrm{C}$, la transmission du parasite doit donc s'effectuer bien plus rapidement pendant l'été. Par contre, il semble qu'à 4-6 ${ }^{\circ} \mathrm{C}$, les larves ne puissent se développer, la transmission du parasite ne se ferait donc pas dans les eaux froides.

\section{BIBLIOGRAPHIE}

BLANC G., 1989. L'Anguillicolose : répartition géographique, étude clinique, mise au point d'une méthode de diagnostic par échotomographie, moyens de lutte. Th. Méd. Vét., Ecole Nationale Vétérinaire de Nantes, $194 \mathrm{p}$.

BLANC G., MARCHAND A., GERARD J. P., 1990. L'Anguillicolose : un nouveau risque pour la filière Anguille. Aquarevue, 28, 27-32. 
BONNEAU S., 1990. Etudes sur le cycle biologique d'Anguillicola crassus Kuwahara, Niimi et Itagaki, 1974, Nématode parasite de la vessie gazeuse des Anguilles. Mémoires de stage, DEA de Parasitologie. Université Montpellier II Sciences et Techniques du Languedoc, $27 \mathrm{p}$.

DE CHARLEROY D., GRISEZ L., THOMAS K., BELPAIRE C., OLLEVIER F., 1990. The life cycle of Anguillicola crassus. Dis. aquat. Org., 8, 77-84.

DE CHARLEROY D., THOMAS K., BELPAIRE C., 1987. Problems concerning the species determination, biology and diagnostical methods of Anguillicola, a swimbladder nematode in the european eel (Anguilla anguilla L.). EIFAC Working Party on Eel, Bristol, U.K., 13-16 April 1987 (7 p.).

HIROSE H., SEKINO T., EGUSA S., 1976. Notes on the egg deposition, larval migration and intermediate host of the nematode Anguillicola crassa parasitic in the swimbladder of eels. Fish. Pathol., 11, 27-31, en Japonais, résumé anglais.

KENNEDY C.R., FITCH D.J., 1990. Colonization, larval survival and epidemiology of the nematode Anguillicola crassus, parasitic in the eel, Anguilla anguilla, in Britain. J. Fish. Biol., 36, 117-131.

PETTER A. J., FONTAINE Y. A., LE BELLE N., 1989. Etude du développement larvaire de Anguillicola crassus (Dracunculoidea, Nematoda) chez un Cyclopidae de la région parisienne. Ann. Parasit. Hum. Comp., 64, 347-355.

PETTER A. J., CASSONE J., LE BELLE N., 1990. Observations sur la biologie des premiers stades larvaires d'Anguillicola crassus, nématode parasite de l'Anguille. Ann. Parasit. Hum. Comp., 65, 28-31.

TESCH F. W., 1977. The eel biology and management of Anguillid eels. Translated by Jennifer Greenwood. English Edition edited by P. H. Greenwood, D. Sc. Chapman and Hall Ltd, Londres, (423 p.). 